\title{
Laparoscopic En Bloc Resection of Colon Cancer with Ovarian Metastasis
}

\author{
Amruta Jaiswal ${ }^{1}$, Shazia Khan², Kuan-Gen Huang ${ }^{3}$
}

\begin{abstract}
Most common primary malignancies linked to Krukenberg tumors are those arising from the gastrointestinal tract. Complete cytoreduction is the key to reduce reoccurrence. We report a case of a 34-year-old woman presented with bilateral Krukenberg tumor with no gastrointestinal symptoms or sign; further preoperative evaluation revealed malignant growth in the sigmoid colon. Laparoscopic total hysterectomy, bilateral salpingo-oophorectomy with anterior colonic resection was performed. Histopathology suggested Krukenberg tumor arising from moderately differentiated adenocarcinoma of the colon with further confirmation on immunohistochemistry. The patient had uneventful recovery with no documented evidence of locoregional reoccurrence till date. This case highlights the importance of thorough preoperative evaluation to detect occult primary in the case of Krukenberg tumor; further, it justifies the use of minimal access surgery for en bloc resection of Krukenberg tumor along with primary colonic malignancy.

Keywords: Colon cancer, En bloc resection, Krukenberg tumor, Laparoscopy.

Journal of South Asian Federation of Obstetrics and Gynaecology (2020): 10.5005/jp-journals-10006-1755
\end{abstract}

\section{INTRODUCTION}

Krukenberg tumors were initially described by Friedrich Ernst Krukenberg in 1896 as uncommon metastatic tumors of the ovary ${ }^{1}$ and reported frequently in previous series ranging from $0.7-6.7 \% .^{2}$ More than $90 \%$ of the Krukenberg tumors are from either gastric or colorectal cancers; however, metastasis from colorectal cancer experiences a better prognosis than those from gastric cancer and benefit more from metastasectomy. ${ }^{2}$ The metastasectomy significantly lengthens overall survival in the patients with primary colorectal or breast cancer, higher KPS score, and optimal metastasectomy. ${ }^{3}$ In most of the cases, primary carcinoma is diagnosed prior to the detection of metastatic ovarian tumors; however, an ovarian mass can sometimes be the initial clinical manifestation of the primary disease. Such cases are often misdiagnosed as primary ovarian cancers. Herein, we report a case of en bloc resection for Krukenberg tumor together with uterus, cervix, and colon. In this case, an accurate diagnosis of metastatic tumors was made following resection of the colonic adenocarcinoma and immunohistochemistry.

\section{Case Description}

A 34-year-old female with the history of intermittent dull aching lower abdominal pain of a three-month duration with no abdominal signs was evaluated outside and referred to us with the diagnosis of bilateral ovarian tumor. Transvaginal ultrasonography showed right and left ovarian tumors of $7.4 \mathrm{~cm}$ and $4.3 \mathrm{~cm}$, respectively, with irregular hyperechoic solid pattern, necrosis, and neovascularization on Doppler suggesting Krukenberg tumor (Fig. 1A). CA-125, CA 15-3, CEA, and CA 19-9 were $15.3 \mathrm{U} / \mathrm{mL}, 5.1 \mathrm{U} / \mathrm{mL}, 1.07 \mathrm{ng} / \mathrm{mL}$ and $17.93 \mathrm{U} / \mathrm{mL}$, respectively. Upper gastrointestinal endoscopy was inconclusive but colonoscopy revealed sigmoid ulceroproliferative growth at $30 \mathrm{~cm}$ from anal verge (Fig. 1B), the biopsy suggested moderately differentiated adenocarcinoma.

Laparoscopy confirmed transmural sigmoid colon growth with puckering along mesocolon without any local adherence and bilateral ovarian tumor (Fig. 2). Laparoscopic total hysterectomy
${ }^{1}$ Department of Obstetrics and Gynecology, Chang Gung Memorial Hospital, Linkou, Kweishan, Taoyuan, Taiwan

${ }^{2}$ Department of Obstetrics and Gynecology, Chang Gung Memorial Hospital, Linkou, Kweishan, Taoyuan, Taiwan; Department of Obstetrics and Gynecology, Army Hospital Research and Referral, Delhi Cantt, New Delhi, India

${ }^{3}$ Department of Obstetrics and Gynecology, Chang Gung Memorial Hospital, Linkou, Kweishan, Taoyuan, Taiwan; Department of Obstetrics and Gynecology, Chang Gung University College of Medicine, Kweishan, Taoyuan, Taiwan

Corresponding Author: Kuan-Gen Huang, Department of Obstetrics and Gynecology, Chang Gung Memorial Hospital, Linkou, Kweishan, Taoyuan, Taiwan; Department of Obstetrics and Gynecology, Chang Gung University College of Medicine, Kweishan, Taoyuan, Taiwan, Phone: +886-3-3281200 ext 8253, e-mail: kghuang@ms57.hinet.net

How to cite this article: Jaiswal A, Khan S, Huang K-G. Laparoscopic En Bloc Resection of Colon Cancer with Ovarian Metastasis. J South Asian Feder Obst Gynae 2020;12(1):45-47.

Source of support: Nil

Conflict of interest: None

with bilateral salpingo-oophorectomy, omentectomy, and lymphadenectomy and peritoneal sampling were performed after anterior resection of the sigmoid colon. Intraoperative frozen section of the right ovary showed metastatic adenocarcinoma with necrosis (AJCC stage T4, N1, M1, R0).

Histopathology of the resected colonic specimen on gross appearance showed a fungating mass with a central ulceration invading serosal surface with circumferential margin of more than $1 \mathrm{~cm}$, myomatous uterine nodule, and right and left ovaries measuring $6.4 \times 5.5 \times 5.1$ and $3.6 \times 3.1 \times 1.9 \mathrm{~cm}$, respectively, with solid heterogeneous yellowish-gray material with necrosis (Fig. 3 ). Microscopically colonic mucosa and ovary revealed moderately differentiated adenocarcinoma. Pericolic lymph node showed metastatic adenocarcinoma. Uterus, cervix, and tubes did not 

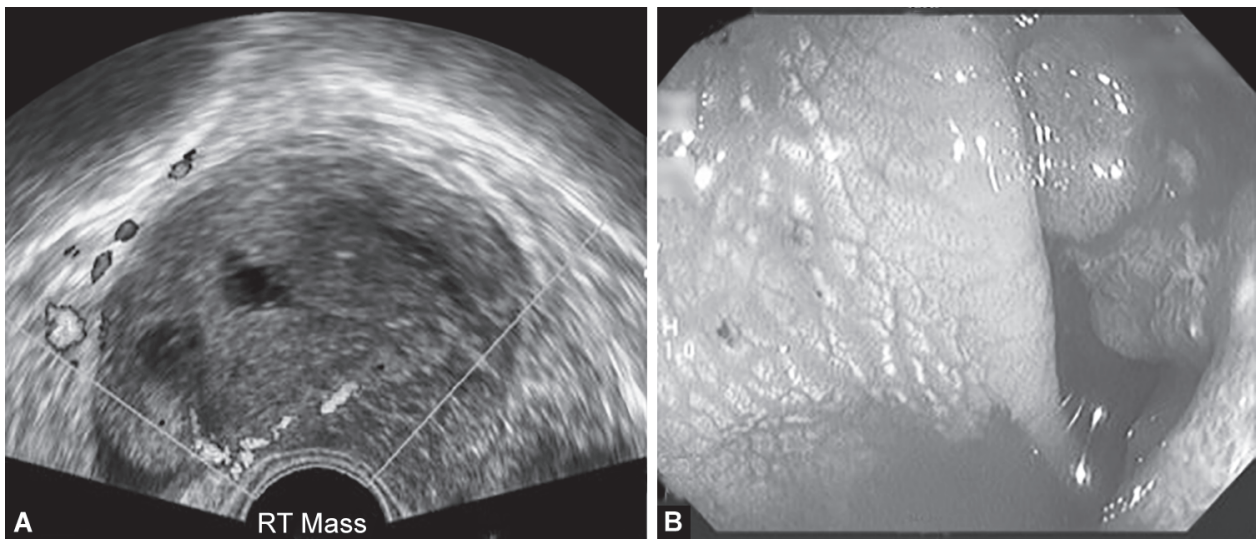

Figs $1 \mathrm{~A}$ and B: (A) Ultrasonography showing right ovarian tumor with hyperechoic solid pattern and neovascularization; (B) Colonoscopy showing sigmoid colon growth
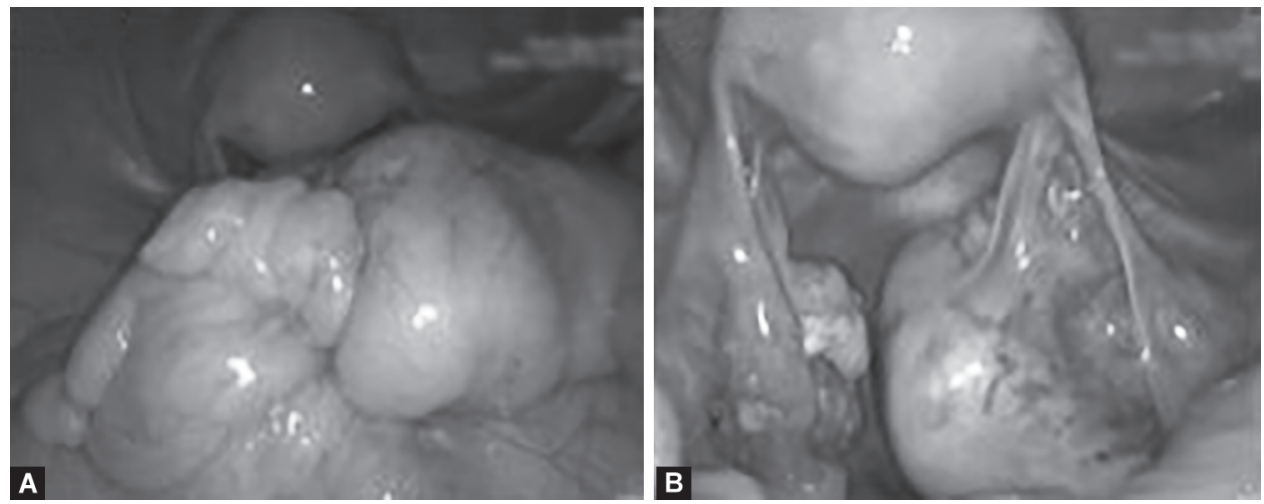

Figs 2A and B: Laparoscopic view: (A) Circumferential involvement of sigmoid colon; (B) Bilateral Krukenberg tumor

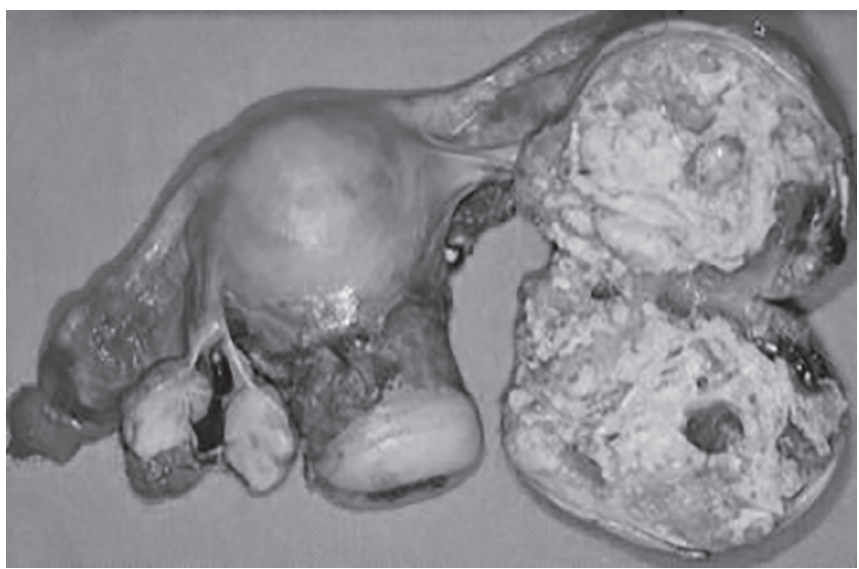

Fig. 3: Cut section of bilateral ovaries showing solid heterogenic tumor with necrosis

show any malignant cells. Peritoneal fluid cytology was negative for malignancy.

Immunohistochemical study (IHC2015-3132) revealed positive CK20 and negative CK7. All of these features confirmed the diagnosis of Krukenberg tumor arising from primary sigmoid colon adenocarcinoma. She had an uneventful recovery and was discharged on the seventh postoperative day with instruction to receive six cycles of chemotherapy with FOLFOX. On follow-up, the patient did not show any locoregional reoccurrence or distant metastases of tumor till date.

\section{Discussion}

Krukenberg tumors constitute $1-2 \%$ of all ovarian neoplasms and most common primary origin are gastrointestinal malignancy, usually presented in younger females with an average age of 45 years. ${ }^{3}$ Some rare cases were seen arising from carcinoma of the breast, small intestine, appendix, gallbladder, pancreas, ampulla of Vater, non-Hodgkin lymphoma, etc. The most common presenting symptoms are abdominal pain, increasing abdominal girth, vomiting, postmenopausal bleeding, weight loss, or rectal bleeding. Our patient presented with vague abdominal pain. Metastatic Krukenberg tumor occurs by three possible routes lymphatic, hematogenous, and transcoelomic. Metastasis from colon cancer can be through retrograde lymphatic spread, and ovaries are among the first organ to be involved. ${ }^{4}$

Transvaginal or transabdominal ultrasound with Doppler is the important tool to differentiate primary ovarian tumor from metastatic ovarian malignancy. Features like bilateral involvement with hyperechoic solid pattern and neovascularization with low impedance flow can suspect ovarian metastases. ${ }^{5}$ In our case, the above features were present which lead us to suspect Krukenberg tumor and we made other investigations to search for primary.

Surgical treatment should be aimed at complete resection of primary tumor with ovarian metastasis and the removal of both ovaries. There is no advantage in preserving any ovarian tissue in case of metastasis as a risk of further tumor involvement is high. Survival rate is more if en bloc resection of tumor and localized metastasis is performed. ${ }^{6}$ Orcutt et al. concluded 
that the use of minimally invasive surgeries in colon cancer resection leads to the improvement in short-term outcomes and decrease in cost of care, without compromising oncologic outcomes. ${ }^{7}$ Reoccurrence after resection of Krukenberg tumor is common; hence, to improve the outcome of the patient, bilateral salpingo-oophorectomy or more aggressive debulking surgery is recommended. ${ }^{8}$

Immunohistochemistry (IHC) results of positive CK20 and negative CK7 strongly suggest ovarian metastasis from colorectal cancers, ${ }^{9}$ which confirmed our diagnosis of Krukenberg tumor secondary to sigmoid colon cancer. ${ }^{10}$

\section{Conclusion}

In conclusion, this case report of synchronous malignancy of Krukenberg tumor with occult primary sigmoid adenocarcinoma emphasizes that preoperative evaluation of the patients with Krukenberg tumor is necessary to avoid misdiagnosing them as primary ovarian tumor. En bloc resection plays a key role to reduce the recurrence rate and improve disease-free survival. As in our case, laparoscopic en bloc removal of Krukenberg tumor improved surgical outcome with added advantage of reduced morbidity and early recovery. Hence, this approach should be considered standard of care in the patients for whom a minimally invasive surgical approach is feasible.

\section{References}

1. Krukenberg F. Ueber das fibrosarcoma ovarii mucocellulare (carcinomatodes). Arch Gynecol Obstet 1896;50(2):287-321.
2. Lu LC, Shao YY, Hsu CH. Metastasectomy of Krukenberg tumors may be associated with survival benefits in patients with metastatic gastric cancer. Anticancer Res 2012;32(8):3397-3401.

3. Rosa F, Marrelli D, Morgagni P. Krukenberg tumors of gastric origin: the rationale of surgical resection and perioperative treatments in a multicenter western experience. World J Surg 2016;40(4):921-928. DOI: 10.1007/s00268-015-3326-8.

4. Jiang R, Tang J, Cheng $X$, et al. Surgical treatment for patients with different origins of Krukenberg tumors: outcomes and prognostic factors. Euro J Surg Oncol (EJSO) 2009;35(1):92-97. DOI: 10.1016/ j.ejso.2008.05.006.

5. Moore RG, Chung M, Granai CO, et al. Incidence of metastasis to the ovaries from nongenital tract primary tumors. Gynecol Oncol 2004;93(1):87-91. DOI: 10.1016/j.ygyno.2003.12.039.

6. Chen CY, Wu YC, Yen MS, et al. The power Doppler velocity index, pulsatility index, and resistive index can assist in making a differential diagnosis of primary ovarian carcinoma and Krukenberg tumors a preliminary study. J Ultrasound Med 2007;26(7):921-926. DOI: 10.7863/jum.2007.26.7.921.

7. Hunter JA, Ryan JA, Schultc P. En bloc resection of colon cancer adherent to other organs. Am J Surg 1987;154(1):49-53. DOI: 10.1016/0002-9610(87)90292-3.

8. Orcutt ST, Marshall CL, Robinson CN, et al. Minimally invasive surgery in colon cancer patients leads to improved short-term outcomes and excellent oncologic results. Am J Surg 2011;202(5):528-531. DOI: 10.1016/j.amjsurg.2011.06.024.

9. Tai DK, Li WH, Cheung MT. Krukenberg tumours of colorectal origin: experience of a tertiary referral centre and review of the literature. Surg Pract 2012;16(2):46-52. DOI: 10.1111/j.1744-1633.2012.00592.x.

10. Lewis MR, Deavers MT, Silva EG, et al. Ovarian involvement by metastatic colorectal adenocarcinoma: still a diagnostic challenge. Am J Surg Pathol 2006;30(2):177-184. DOI: 10.1097/01.pas.00001764 36.26821.8a. 